\title{
CMEARTICLE \\ Clinics in diagnostic imaging (151)
}

Andrew Yu Keat Khor ${ }^{1}$, BEng, Steven Bak Siew Wong ${ }^{2}$, MBChB, MMed

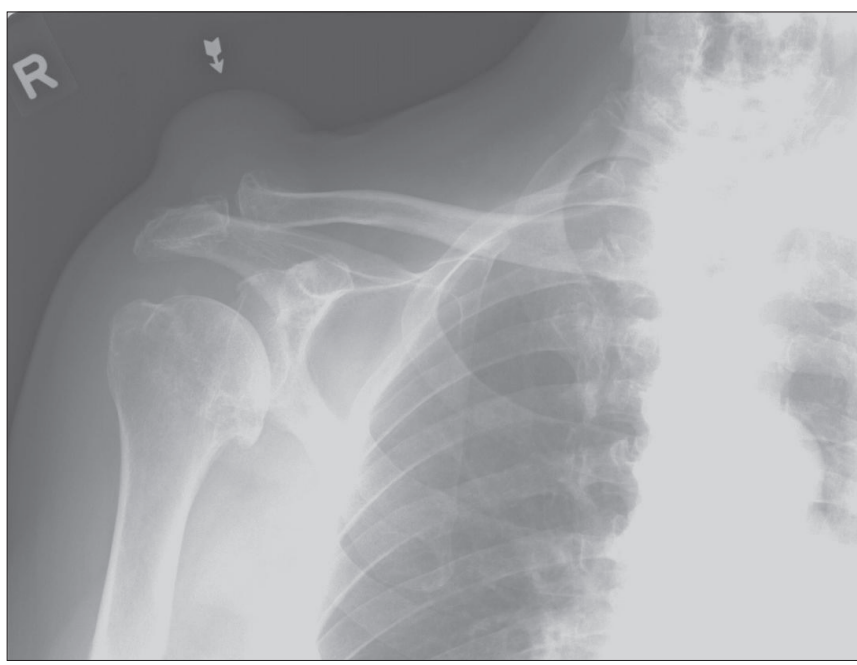

Fig. 1 Frontal radiographic view of the right shoulder joint.
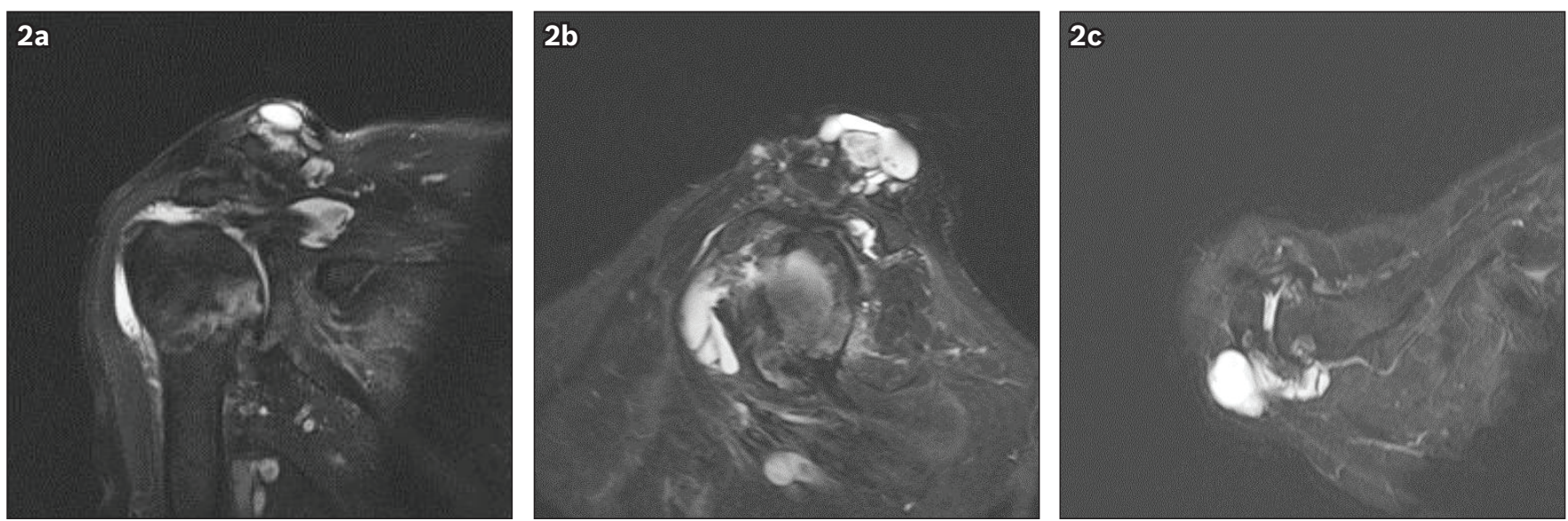

Fig. 2 (a) Coronal, (b) sagittal and (c) axial T2-W turbo inversion recovery magnitude MR images of the right shoulder joint.

\section{CASE PRESENTATION}

An 82-year-old man presented with neck pain, right upper limb radiculopathy and right shoulder pain. His medical history was significant for multiple comorbidities, including osteoarthritis of the left hip joint and both knee joints, as well as cervical degenerative disc disease. Relevant surgical history included right total knee replacement arthroplasty, left total hip replacement arthroplasty and C3-C6 cervical laminectomy. The patient had previously worked as a manual labourer, but presently had difficulty moving his upper limbs.

On physical examination, we noted a soft lump over his right shoulder joint. The lump was compressible, non-mobile and non-tender. Although the patient could not remember when the lump had first appeared, he noted that it had slowly enlarged over the past two years. He also had reduced ipsilateral shoulder movements, particularly in abduction, but showed no painful arc sign. Plain radiographs of the right shoulder joint (Fig. 1), obtained at the clinic visit, were compared with that taken two years prior. Based on the radiographic findings, magnetic resonance (MR) imaging of the right shoulder joint (Fig. 2) was requested. What do the images (Figs. 1 \& 2) show? What is the final diagnosis and what are the underlying conditions?

${ }^{1}$ Duke-NUS Graduate Medical School, ${ }^{2}$ Department of Diagnostic Radiology, Singapore General Hospital, Singapore

Correspondence: Dr Steven Wong Bak Siew, Consultant Radiologist, Department of Diagnostic Radiology, Singapore General Hospital, Outram Road, Singapore 169608. steven.wong@sgh.com.sg 

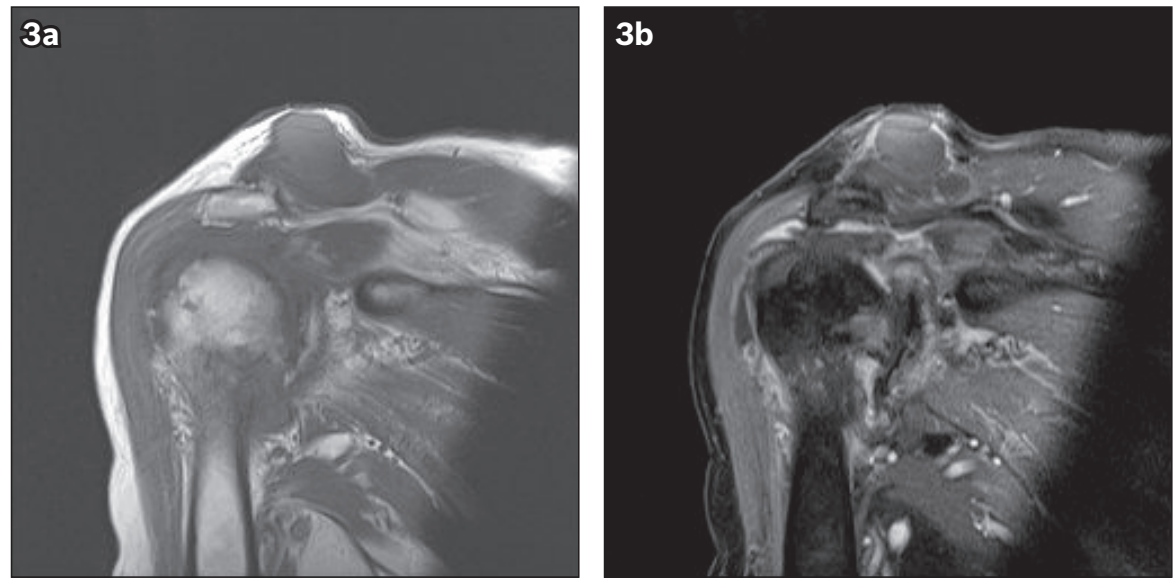

Fig. 3 (a) Pre- and (b) post-intravenous contrast-enhanced coronal T1-W turbo spin echo images of the right shoulder joint.

\section{DIAGNOSIS}

Acromioclavicular joint geyser sign with chronic full-thickness supraspinatus tendon (SST) tear.

\section{IMAGE INTERPRETATION}

The plain radiograph (Fig. 1) of the right shoulder joint shows a soft tissue density mass over the osteoarthritic right acromioclavicular (AC) joint. The soft tissue mass has a fairly smooth outline and a homogeneous appearance with no underlying bony destruction. Osteoarthritic changes are noted at both the right $\mathrm{AC}$ and the glenohumeral joints, including marginal osteophytes, articular surface irregularity and joint space narrowing. The right humeral head shows slight caudal migration relative to the glenoid process. Non-arthrographic MR images (Figs. 2a-c) of the right shoulder joint reveal a full-thickness tear of the SST, with the remnant tendon retracted to a position just proximal to the glenoid process. Synovial fluid is seen in the subacromial space, communicating with the right $\mathrm{AC}$ joint, and within the mass over the right $\mathrm{AC}$ joint. The mass shows T2-weighted hyperintense and T1-weighted hypointense signal characteristics of fluid. The multilobulated and multiseptated cystic mass can be traced to the posterior superior aspect of the right AC joint and the subacromial bursal effusion. No enhancement with intravenous contrast medium is detected (Fig. 3). This is the classic geyser sign of a large cyst overlying and communicating with the $\mathrm{AC}$ joint in the setting of $\mathrm{a}$ torn SST.

\section{CLINICAL COURSE}

Owing to the benign nature of the cystic mass and the marked, chronically retracted status of the patient's right SST, no treatment was instigated despite the reduced range of movement in his right shoulder joint.

\section{DISCUSSION}

The geyser sign is an infrequently encountered imaging sign that was originally described on conventional fluoroscopy-guided shoulder arthrography. With the increased use of MR imaging for the evaluation of shoulder joint disorders, the ability to recognise the presence of the geyser sign on both conventional and arthrographic MR images is essential. The sign derives its name from its geyser-like appearance. On imaging, owing to a full-thickness SST tear, synovial fluid or intra-articular contrast extravasates from the glenohumeral joint into the subacromial bursa. The fluid, which then passes through the AC joint into an overlying cystic mass, is said to resemble a geyser spouting upwards.

Originally described by Craig in 1984, ${ }^{(1)}$ no other study has documented the prevalence of the geyser sign in the setting of a chronic SST tear to date. In an analysis of nine cases of AC joint cyst, Tshering Vogel et al reported positive geyser sign, with conventional shoulder arthrograms that showed SST tears, in three cases. ${ }^{(2)} \mathrm{A}$ study by Chalian et al reported that only 1 out of $56(1.8 \%)$ patients who had MR imaging post shoulder trauma showed findings suggestive of a geyser sign. ${ }^{(3)}$

The signs and symptoms of SST disease are highly variable, ranging from asymptomatic patients with a full range of shoulder movements to those who are unable to lift their arms due to constant shoulder pain. ${ }^{(4)}$ This may depend on several factors, including the degree of the SST tear, the patient's age, and the presence of other comorbidities such as rheumatoid arthritis, osteoarthritis and septic arthritis. While the supraspinatus muscle works with the deltoid muscle during abduction of the arm, patients with massive SST tears are often unable to lift their arms fully due to pain or weakness. Physical examination tests such as the drop arm test and Jobe's test, as well as isometric strength testing of the supraspinatus, may help in the evaluation of disease severity.

The causes of SST tears are multifactorial, with mechanical impingement and joint degeneration postulated as possible contributory factors. Craig proposed that as an SST tear progresses, the loss of external rotation power results in relatively unopposed internal rotation, causing the arc of elevation to move medially, centring beneath the coracoacromial ligament and AC joint, exposing the remaining intact tendon to further impingement. ${ }^{(1)}$ With a very large tendon 

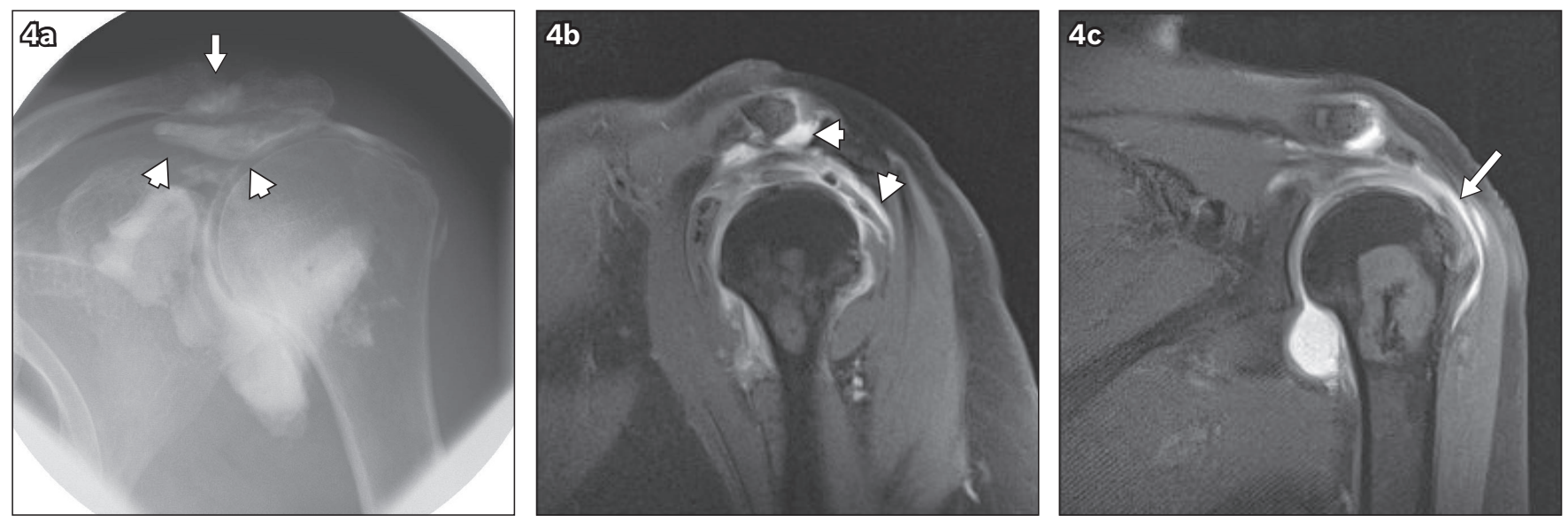

Fig. 4 (a) Fluoroscopic spot image from arthrography of the shoulder of a 48-year-old female patient shows entry of the contrast mixture into the left acromioclavicular joint (arrow). Note the large quantity of contrast mixture within the subacromial bursa (arrowheads). (b) Sagittal and (c) coronal T1-W turbo spin echo images from subsequent MR arthrography in the same patient confirm the presence of contrast mixture within the acromioclavicular joint and subacromial bursa (arrowheads). Small, focal, minimally retracted full-thickness tears of the left supraspinatus tendon are seen close to the greater tuberosity insertion site (arrow).

tear, repeated trauma may lead to gradual erosion of the inferior/deep AC joint capsule, resulting in the production of the geyser sign on arthrography. ${ }^{(2)}$

Cystic masses over the AC joint can occur in the setting of a massive rotator cuff tear. These cysts, which are usually painless and enlarge progressively, can be mistaken for malignancy. ${ }^{(5)}$ The mass is usually firm but compressible, non-mobile and non-tender to touch. The size of the cyst varies and is unrelated to the size of the SST tear. While the growth of the cyst is typically insidious and progressive, it may occasionally regress in size.

The actual pathogenesis of AC joint cysts is not fully understood. They are thought to occur when the synovial fluid in the glenohumeral joint escapes via the full-thickness SST tear into the subacromial bursa, and then decompresses across an osteoarthritic AC joint. The increased intra-articular fluid pressure eventually causes distension, outpouching, and possibly, rupture of the superior capsule of the AC joint, leading to the formation of a cystic mass. ${ }^{(6)}$ Plain radiograph evaluations are nonspecific, thus requiring a high index of suspicion and prior knowledge of existing SST tears. On plain radiography, a focal soft tissue swelling may be seen over the superior/superficial aspect of the AC joint, isodense to adjacent soft tissue. There may be associated AC joint osteoarthritis with joint widening and marginal osteophytes. This was the case with our patient. Calcification within the soft tissue mass is seldom seen. With conventional shoulder arthrography, leakage of contrast medium from the glenohumeral joint into the subacromial bursa will outline the passage through the AC joint and fill the centre of the cyst, leading to the classical geyser sign. This is illustrated in the arthrography (Fig. 4a) and MR images (Figs. 4b \& c) of another case.

Ultrasonography (US) may be requested to evaluate the shoulder lump or rotator cuff. On US, cysts over the AC joint appear anechoic or hypoechoic. One should look out for communication with the $\mathrm{AC}$ joint and confirm the presence of an SST tear. Communication with a subacromial bursa related to the SST tear may not be demonstrable due to the presence of bone. SST tears, readily detected during US, are seen as a hypoechoeic defect within the tendon and as a dip in the normal convex contour. The tendon may be retracted in the case of full-thickness complete tears. Thus, a high index of suspicion is required when evaluating using US. The effectiveness of US is severely reduced due to the inability of the US beam to penetrate the bony cortex for evaluation of the SST deep to the acromion.

There have been few reported cases of geyser signs on computed tomography (CT), likely because CT is infrequently used to evaluate lumps in the shoulder region. While CT can detect full-thickness SST tears, partial tears may not be evident due to the inherent poor tissue resolution of the tendons on $\mathrm{CT}^{\left({ }^{(7)}\right.}$ As such, a negative $\mathrm{CT}$ does not rule out a possible tendon tear, and further evaluation with US or MR imaging may be warranted. Secondary signs on CT include retracted SST, the presence of AC joint osteophytes or subacromial bony spurs. The cyst over the AC joint may show a homogeneous hypodense appearance with fluid attenuation values. Calcification may be seen at the margins of the cyst.

Mohana-Borges et al have described the geyser sign on postoperative MR imaging secondary to injury of the acromial undersurface during surgery. ${ }^{(8)} \mathrm{MR}$ imaging can accurately depict the cystic nature of the mass over the AC joint. On T2-weighted sequences, the geyser sign is seen as hyperintense synovial fluid extending across the subacromial space and through the AC joint into the overlying cyst. ${ }^{(6)} \mathrm{A}$ membranous capsule, if present, is depicted as a thin hypointense surrounding layer on T1- and T2-weighted sequences. Internal septations or multiple lobulations are more clearly shown on MR imaging. Rupture of the AC joint cyst may be seen as free fluid in the surrounding soft tissue planes. Additionally, MR imaging may demonstrate associated SST tears, seen as discontinuity in the tendon at, or proximal to, the insertion site at the humeral 
greater tuberosity. The tendon defect/tear site may be filled with T2-weighted hyperintense synovial fluid. Note that MR arthrography is not commonly performed for the evaluation of shoulder masses. In the absence of inflammation, enhancement of the capsule of the AC joint cyst is rarely seen, while the fluid within the AC joint cyst generally shows no enhancement. Intravenous contrast medium may, however, indicate the presence of a leak or rupture of the AC joint cyst.

\section{CONCLUSION}

When present, the geyser sign can provide invaluable information regarding the integrity of the SST. Full-thickness SST tear, complete or partial, may not be suspected prior to imaging examination. Such an examination can help in prognostication and the evaluation of treatment options, as the geyser sign usually results from a chronic, and possibly large, full-thickness SST tear. ${ }^{(1)}$ The demonstration of the geyser sign during the assessment of a shoulder lump for presence of malignancy indicates a benign process. ${ }^{(6)}$

ABSTRACT An 82-year-old man presented with neck pain, right upper limb radiculopathy and right shoulder pain. Physical examination revealed a soft lump over the right shoulder joint, as well as reduced range of shoulder movements. On magnetic resonance imaging, the soft lump was shown to be a cystic mass over the acromioclavicular joint and was related to a full-thickness supraspinatus tendon tear. This is the classic geyser sign. The pathophysiology and clinical features of the geyser sign, and its imaging features with various imaging modalities, are discussed.

Keywords: acromioclavicular joint cyst, geyser sign, magnetic resonance imaging, rotator cuff tears

\section{REFERENCES}

1. Craig EV. The geyser sign and torn rotator cuff: clinical significance and pathomechanics. Clin Orthop Relat Res 1984; (191):213-5.

2. Tshering Vogel DW, Steinbach LS, Hertel R, et al. Acromioclavicular joint cyst: nine cases of a pseudotumor of the shoulder. Skeletal Radiol 2005; 34:260-5.

3. Chalian M, Soldatos T, Faridian-Aragh N, et al. MR evaluation of synovial injury in shoulder trauma. Emerg Radiol 2011; 18:395-402.

4. McFarland EG, Garzon-Muvdi J, Jia X, Desai P, Petersen SA. Clinical and diagnostic tests for shoulder disorders: a critical review. Br J Sports Med 2010; 44:328-32.

5. Murena L, D'angelo F, Falvo DA, Vulcano E. Surgical treatment of an aseptic fistulized acromioclavicular joint cyst: a case report and review of the literature. Cases J 2009; 2:8388.

6. Cooper HJ, Milillo R, Klein DA, DiFelice GS. The MRI geyser sign: acromioclavicular joint cysts in the setting of a chronic rotator cuff tear. Am J Orthop (Belle Mead NJ) 2011; 40:E118-21.

7. Lecouvet FE, Simoni P, Koutaïssoff $S$, et al. Multidetector spiral CT arthrography of the shoulder. Clinical applications and limits, with MR arthrography and arthroscopic correlations. Eur J Radiol 2008; 68:120-36.

8. Mohana-Borges AV, Chung CB, Resnick D. MR imaging and MR arthrography of the postoperative shoulder: spectrum of normal and abnormal findings. Radiographics 2004; 24:69-85. 


\section{SINGAPORE MEDICAL COUNCIL CATEGORY 3B CME PROGRAMME} (Code SMJ 201402A)

Question 1. The geyser sign is related to:

(a) Subacromial bursa containing synovial fluid.

(b) Acromioclavicular joint space widening and degeneration.

(c) Full-thickness tears of the supraspinatus tendon.

(d) A malignant lesion eroding the lateral clavicle.

Question 2. The geyser sign is associated with:

(a) A recent acute injury to the shoulder joint.

(b) A bursal-sided partial-thickness tear of the supraspinatus tendon.

(c) A better prognosis after surgical management.

(d) Defects or injury to the acromioclavicular joint capsule.

Question 3. Regarding the imaging aspects of the geyser sign:

(a) It is diagnosable on plain radiography.

(b) Intra-articular contrast medium (arthrography) is not required to visualise the geyser sign on magnetic resonance (MR) imaging.

(c) Computed tomography (CT) is superior to ultrasonography (US) in evaluating the presence or absence of a geyser sign.

(d) MR imaging is less sensitive if no intravenous contrast medium is administered.

Question 4. Regarding the supraspinatus tendon:

(a) CT and US are equally effective in detecting supraspinatus tendon tears.

(b) Full-thickness supraspinatus tendon tears frequently present with the geyser sign.

(c) Tears are easily detected on conventional arthrography.

(d) Tears of the tendon are seen as hyperechoeic lesions on US.

Question 5. Regarding the geyser sign:

(a) It is commonly detected after trauma to the shoulder joint.

(b) It differentiates a benign lesion from a malignant one.

(c) It is of little relevance to the surgical treatment of the patient.

(d) Degenerative changes in the acromioclavicular joint are a prerequisite.

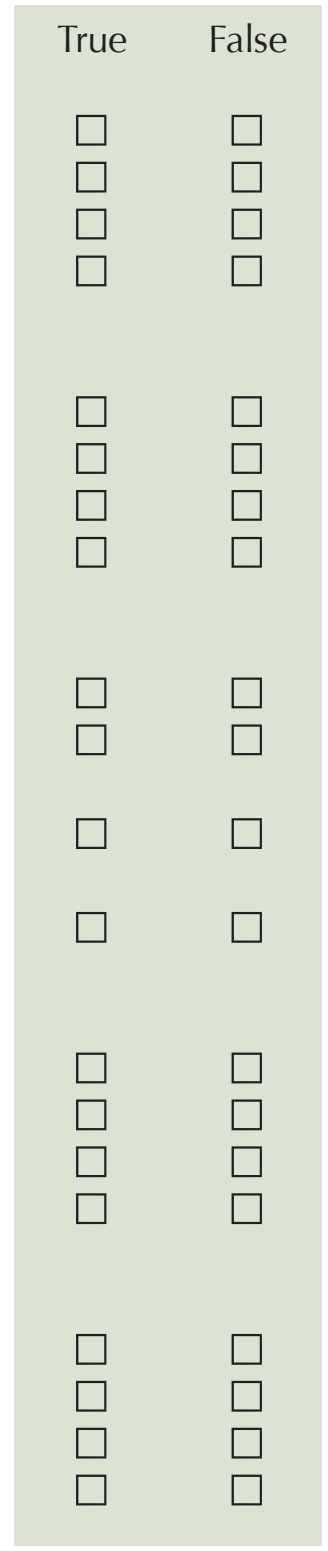

\section{Doctor's particulars:}

Name in full

MCR number

Specialty:

Email address

\footnotetext{
SUBMISSION INSTRUCTIONS:

(1) Log on at the SMJ website: http://www.sma.org.sg/publications/smjcurrentissue.aspx and select the appropriate set of questions. (2) Provide your name, email address and MCR number. (3) Select your answers and click "Submit".

RESULTS:

(1) Answers will be published in the SMJ April 2014 issue. (2) The MCR numbers of successful candidates will be posted online at the SMJ website by 25 March 2014. (3) Passing mark is $60 \%$. No mark will be deducted for incorrect answers. (4) The SMJ editorial office will submit the list of successful candidates to the Singapore Medical Council. (5) One CME point is awarded for successful candidates.

Deadline for submission: (February 2014 SMJ 3B CME programme): 12 noon, 18 March 2014.
} 